\title{
Detección y caracterización molecular de virus respiratorios causantes de infección respiratoria aguda en población adulta
}

\author{
Dylan Lucía Díaz-Chiguer" *, Rocío Tirado-Mendoza², Adrián Márquez-Navarro³, \\ Javier Rolando Ambrosio-Hernández², Ismael Ruiz-Fraga1', Ricardo Ezequiel Aguilar-Vargas', \\ José Manuel Lira-Martínez² y Julio César López-Valdés4
}

${ }^{1}$ Dirección Médica, Instituto de Seguridad y Servicios Sociales de los Trabajadores del Estado, Instituto de Seguridad y Servicios Sociales de los Trabajadores del Estado, Ciudad de México; ${ }^{2}$ Departamento de Microbiología y Parasitología, Medicina UNAM, Ciudad de México; ${ }^{3}$ Departamento de Medicamentos Nuevos y Genéricos, Comisión Federal para la Protección contra Riesgos Sanitarios, Ciudad de México; ${ }^{4}$ Facultad de Medicina de Tampico, Universidad Autónoma de Tamaulipas Dr. Alberto Romo Caballero, Tampico, Tamaulipas. México

\section{Resumen}

Introducción: Las infecciones respiratorias agudas son una de las principales causas de morbimortalidad en adultos mayores y pacientes con enfermedades crónicas. Dentro de los agentes etiológicos responsables se encuentran los virus respiratorios humanos, tales como: virus sincitial respiratorio, virus parainfluenza y metapneumovirus. Objetivo: Realizar un estudio de diagnóstico diferencial de virus respiratorios que circulan y cocirculan en una población adulta. Método: Se realizó un estudio tipo piloto en pacientes mayores de 18 años, que presentaron signos y síntomas sugestivos de infección respiratoria aguda y cuyo cuadro clínico no sobrepasara los 15 días de evolución; se realizaron ensayos de reacción en cadena de la polimerasa de punto final con el uso de oligonucleótidos específicos para el diagnóstico molecular. Resultados: Se tipificaron 72 especímenes de pacientes con una edad de $51.33 \pm 19.33$ años, con predominio del sexo femenino (4.5:1); originarios en su totalidad de la Ciudad de México; solo 22 fueron positivos para virus respiratorios, siendo en su mayoría infecciones por metapneumovirus. Conclusiones: El conocimiento de las cepas virales circulantes en la población permitirá determinar cambios que puedan declarar una alerta epidemiológica llevando a la mejor toma de decisiones en beneficio de los pacientes.

PALABRAS CLAVE: Infecciones respiratorias agudas. Virus respiratorios. Diagnóstico molecular.

\begin{abstract}
Introduction: Acute respiratory infections are one of the main causes of morbidity and mortality in older adults and patients with chronic diseases. Among the responsible etiological agents are human respiratory viruses, such as: respiratory syncytial virus, parainfluenza virus and metapneumovirus. Objective: To carry out a differential diagnostic study of respiratory viruses circulating and co-circulating in an adult population. Methods: A pilot study was conducted in patients older than 18 years, who presented signs and symptoms suggestive of acute respiratory infection and whose clinical picture did not exceed 15 days of evolution; end-point polymerase chain reaction assays were performed with the use of specific oligonucleotides for molecular diagnosis. Results: 72 specimens of patients with an age of $51.33 \pm 19.33$ years, with a predominance of females (4.5:1); original inhabitants of Mexico City; only 22 were positive for respiratory viruses, being mostly metapneumovirus infections. Conclusions: The knowledge of the circulating viral strains in the population will allow to determine changes that can declare an epidemiological alert leading to the best decision making for the benefit of the patients.
\end{abstract}

KEY WORDS: Acute respiratory infections. Respiratory viruses. Molecular diagnosis.

Correspondencia:

*Dylan Lucía Díaz-Chiguer

E-mail: dra.dylandiaz@outlook.com
Fecha de recpción: 06-03-2019

Fecha de aceptación: 19-03-2019

DOI: 10.24875/GMM.19005138
Gac Med Mex. 2019;155(Suppl 1):S16-S21

Disponible en PubMed www.gacetamedicademexico.com 


\section{Introducción}

Las infecciones respiratorias agudas (IRA) son la entidad nosológica más frecuente durante la vida del ser humano. La Organización Mundial de la Salud (OMS) reconoce que algunas IRA causan brotes a gran escala, con alta morbilidad y mortalidad; por lo cual constituyen una emergencia para la salud pública'. En México, en el 2017, a través de la Secretaría de Salud se reportaron más de 26 millones de casos nuevos; aproximadamente 1.6 millones de estos correspondieron a población derechohabiente del Instituto de Seguridad y Servicios Sociales de los Trabajadores del Estado (ISSSTE)².

Aunque la mayoría de las infecciones respiratorias solo afectan al tracto respiratorio superior (catarro común, rinitis y faringoamigdalitis), se estima que alrededor del $5 \%$ de las infecciones puede afectar al tracto respiratorio inferior y producir enfermedades que puedan requerir ingreso hospitalario (bronquitis, bronquiolitis y neumonía) ${ }^{3}$.

En la población infantil, los virus respiratorios (VR) son la causa más común de insuficiencia respiratoria grave $^{4}$. Asimismo, estudios recientes han demostrado que las cifras, tanto de morbilidad como de mortalidad, relacionadas con estas infecciones ha incrementado para la población adulta y ancianos inmunocomprometidos o con afección cardiopulmonar, ${ }^{5,6}$.

Entre los VR que se han reconocido como agentes causales de IRA se encuentran los virus influenza $A$ y B; parainfluenza 1, 2, 3 y 4 (PIV 1-4h), metapneumovirus humano (MPVh), virus sincitial respiratorio humano (VSRh), rinovirus y enterovirus. En su mayoría, son clasificados dentro de la familia Paramixoviridae, género pneumovirus (VSRh y MPVh) y paramixovirus (PIV 1-3h) ${ }^{7,8}$, son altamente contagiosos y responsables de epidemias anuales, principalmente durante el periodo invernal ${ }^{9}$. Ahora bien, en el caso del VSRh se estima que es responsable 33.8 millones de IRA; así como 3.4 millones de admisiones hospitalarias y 250,000 muertes por año. Se ha asociado con el $70 \%$ de bronquiolitis y $25 \%$ de neumonía como complicaciones severas. En tanto que el MPVh y el PIV 1-3h son responsables del $5-15 \%$ de las IRA y del $2-17 \%$ de las admisiones hospitalarias'. Cabe mencionar que el MPVh es considerado un virus emergente y muestra una alta prevalencia en pacientes pediátricos hospitalizados con bronconeumonía y en adultos 5,6 .
A pesar de la importancia evidenciada ante los datos ya mencionados, aún no se cuenta con un método diagnóstico confiable y cuya realización sea sistemática para la pronta detección de VSRh, MPVh y PIV 1-3 h.

Las herramientas actuales para el diagnóstico de las IRA (serológicas, aislamientos de los virus en cultivos celulares e inmunofluorescencia) son de baja sensibilidad. De acuerdo con lo anterior, se considera de gran importancia desarrollar métodos de diagnóstico molecular que ofrezcan una mayor sensibilidad y especificidad en comparación con los métodos descritos previamente. Según esto, se realizó un estudio que tuvo como objetivo diagnosticar y caracterizar por técnicas moleculares de reacción en cadena de la polimerasa (PCR) los VR que circulan y cocirculan en una población adulta diagnosticada con IRA de acuerdo con los criterios de la Guía de Práctica Clínica ${ }^{10,11}$.

\section{Métodos}

Se realizó un estudio transversal tipo piloto dentro de la Clínica de Especialidades Indianilla del ISSSTE en la Ciudad de México en el periodo comprendido entre agosto de 2016 y marzo de 2017. Se incluyeron pacientes mayores de 18 años, que presentaron signos y síntomas sugestivos de IRA y cuyo cuadro clínico no sobrepasara los 15 días de evolución. En contraste, fueron excluidos los pacientes que mostraron signos y síntomas compatibles con infección bacteriana, quienes recibieron antibioticoterapia o antivirales durante los últimos 7 días; así como aquellos que mostraban criterios de severidad a la exploración física (p. ej., disnea de reposo, cianosis periférica, taquicardia, taquipnea) y quienes sus signos y síntomas pudieron ser atribuidos a otro diagnóstico: rinitis alérgica, sinusitis crónica.

Es de señalar que dicha clínica atiende anualmente aproximadamente 26,000 pacientes en atención médica continua; así como 92,000 (aproximadamente) consultas de atención especializada (p. ej., otorrinolaringología, neumología e inhaloterapia) procedentes de 18 clínicas de medicina familiar propias a la Ciudad de México y al Estado de México.

Para realizar la investigación se obtuvo autorización por parte del Comité de Investigación, así como del Comité de Ética pertenecientes a la institución; en el que se incluyeron las condiciones de bioseguridad para manejo de las muestras biológicas y/o microorganismos patógenos aislados de acuerdo a los protocolos establecidos por la NOM-087SEMARMAT-SSA1-2002, 
Tabla 1. Relación de oligonucleótidos y secuencias de los genes de F, GA y MLGB de VSRh, N y L de MPVh y HN de PIV $2 \mathrm{~h}$

\begin{tabular}{|c|c|c|c|c|c|}
\hline Gen/sentido & Secuencia & Gen & $\begin{array}{l}\text { Tamaño de } \\
\text { fragmento }(\mathrm{pb})\end{array}$ & $\begin{array}{l}\text { Región del } \\
\text { gen }\end{array}$ & $\begin{array}{l}\text { Temperatura de } \\
\text { alineamiento }\left({ }^{\circ} \mathrm{C}\right)\end{array}$ \\
\hline \multicolumn{6}{|c|}{ Oligonucleótidos para VSRh } \\
\hline $\mathrm{Ff}$ & 5'ATG'AACAGTTTAACATTACCAAGTGA3' & \multirow[t]{2}{*}{$\mathrm{F}$} & \multirow[t]{2}{*}{193} & \multirow[t]{2}{*}{$1127-1320$} & \multirow[t]{2}{*}{56} \\
\hline $\mathrm{Fr}$ & 5'CCACGATTTTTATTGGATGCTG3' & & & & \\
\hline Gf & 5'CCCAACATACCTCACT3' & \multirow[t]{2}{*}{$G(A)$} & \multirow[t]{2}{*}{549} & \multirow[t]{2}{*}{$275-524$} & \multirow[t]{2}{*}{55} \\
\hline Gr & 5'GAGGAGGTTGAGTGGAAG3' & & & & \\
\hline MLf & 5'CATGCCAAACACAAGAATCAAC3' & \multirow[t]{2}{*}{$\mathrm{ML}(\mathrm{B})$} & \multirow[t]{2}{*}{106} & \multirow[t]{2}{*}{$102-204$} & \multirow[t]{2}{*}{58} \\
\hline MLr & 5'ATTCATCATCTCTGCCAATCAC3' & & & & \\
\hline \multicolumn{6}{|c|}{ Oligonucleótidos para MPVh } \\
\hline $\mathrm{Nf}$ & 5'-CAACAGCAGTGACACCCTC-3' & \multirow[t]{2}{*}{$\mathrm{N}$} & \multirow[t]{2}{*}{599} & \multirow[t]{2}{*}{$148-747$} & \multirow[t]{2}{*}{60} \\
\hline $\mathrm{Nr}$ & 3'-ACTCATACCGTTTCGTAA-5' & & & & \\
\hline Lf & 5'-GCCATAGCCCAAACCATA- 3' & \multirow[t]{2}{*}{$\mathrm{L}$} & \multirow[t]{2}{*}{447} & \multirow[t]{2}{*}{$8920-9367$} & \multirow[t]{2}{*}{66} \\
\hline $\mathrm{Lr}$ & 3’-CCCTGTAACGACTAGACT-5' & & & & \\
\hline \multicolumn{6}{|c|}{ Oligonucleótidos para PIV 2h } \\
\hline $\mathrm{HNf}$ & 5'-GACGCCTAAATATGGACCTCTC-3' & \multirow[t]{2}{*}{$\mathrm{HN}$} & \multirow[t]{2}{*}{279} & \multirow[t]{2}{*}{$525-826$} & \multirow[t]{2}{*}{68.8} \\
\hline $\mathrm{HNr}$ & 5'-CACGTCTGGTCTTCCATCTTT-3' & & & & \\
\hline
\end{tabular}

Protección Ambiental Salud Ambiental Residuos Peligrosos Biológicos Infecciosos Clasificación y Especialización de Manejo, que se generen en establecimientos que prestan atención médica.

\section{Recolección de datos}

Se aplicó un cuestionario para conocer datos concordantes con IRA, tales como: temperatura mayor a $37.5^{\circ} \mathrm{C}$, tos con o sin expectoración, odinofagia, disfonía, malestar general, estornudos, artralgias, mialgias, disnea, amigdalitis, estertores, sibilancias, rinorrea, entre otros. De igual manera, se realizó una historia clínica con la finalidad de verificar la inexistencia de automedicación mediante antibióticos o antivirales durante 7 días previos al estudio; posteriormente, se procedió a la toma del espécimen por hisopado faríngeo y tonsilar. La recolección de datos fue manual, diaria, con posterior revisión del correcto llenado de cada uno de los instrumentos.

\section{Método para la obtención de la muestra}

Previo a la toma de la muestra, se aseguró que los candidatos no se hubiesen realizado aseo bucal antes de asistir a la clínica. En condiciones de esterilidad, con un abatelenguas, se sujetó la lengua del paciente, y se frotó con firmeza la pared posterior de la orofaringe con un hisopo de algodón seco y estéril; se intentó obtener la mayor cantidad de células. El hisopo con el espécimen se colocó en un tubo de plástico estéril que contenía medio de transporte (medio de cultivo DMEM suplementado con albúmina sérica bovina al $5 \%$, glutamina $1 \%$ y penicilina $1 \%$ ), el cual se mantuvo a $4^{\circ} \mathrm{C}$ hasta que se procesaron para aislamiento viral y genotipificación. Una vez llevado a cabo el aislamiento se preservaron a $-70^{\circ} \mathrm{C}$.

\section{Genotipificación de los virus respiratorios}

Con el fin de identificar y confirmar los VR en los especímenes, así como en los controles positivos, se realizaron ensayos de reacción en cadena de la polimerasa de punto final (RT-PCR) con el uso de oligonucleótidos específicos diseñados ex profeso para cada uno de los virus en estudio (Tabla 1); para VSRh, los genes $F$ (fusión) y para la subtipificación de los subgrupos $A$ o $B$ se amplificó el gen $G$ (unión) o ML respectivamente; para MPVh, los genes $N$ (nucleocápside) y $L$ (ARN polimerasa) mientras que para PIV $2 \mathrm{~h}$, el gen $\mathrm{HN}$ (hemaglutinina-neuraminidasa). 
Para el diseño de los oligonucleótidos se utilizó el programa OligoAnalyzer ${ }^{\circledR}$ V 3.1 y tuvo como base la secuencia consenso construida a partir de secuencias completas de aislados virales reportados en Genbank de los cuatro subtipos: A1 (KC588902.1), A2 (FJ168779.1), B1 (AY525843.1) y B2 (FJ168778.1) de MPVh. Para VSRh, subgrupo A cepa Long (NC_001781.1 y AY911262.1); VSRh subgrupo B cepa 18537 (GenBank: D00397.1) y para hPIV (AB367954). Posteriormente los alineamientos se llevaron a cabo con el programa Clustal Omega EMBL-EBI. De igual manera, se examinó la formación de dímeros o estructuras secundarias y alineamientos mediante BLAST con la base de datos de secuencias nucleotídicas de $\mathrm{NCBI}$, con lo cual se descartaron los alineamientos con genes de otros organismos o con regiones. Los marcadores moleculares (oligonucleótidos) diseñados en el laboratorio para llevar a cabo el diagnóstico diferencial viral (VSRh, MPVh y/o PIV 2h), permiten incluir los tipos virales y las variantes virales que estén en circulación en la población en estudio.

\section{Análisis estadístico}

Los datos obtenidos fueron analizados con el programa SPSS ${ }^{\circledR}$ Statistics (Versión 19) utilizando la prueba de $\chi^{2}$ (chi cuadrada) para variables cuantitativas y se consideró significancia estadística cuando se obtenía una $p<0.05$. Se consideraron como «elementos perdidos" aquellas muestras que no cumplieron con los datos requeridos y/o aquellas muestras clínicas que, bajo nuestro criterio de selección para aislamiento viral, resultaron contaminadas con bacterias.

\section{Resultados}

\section{Etapa I. Caracterización de los virus}

Durante el periodo de estudio se obtuvieron 72 especímenes mediante exudado faríngeo de pacientes con diagnóstico clínico de IRA, de los cuales únicamente 22 (30.55\%) cumplieron con los criterios de inclusión e historia clínica completa que apoyara su información epidemiológica. Los datos demográficos mostraron una media de edad de 51.33 (DE \pm 19.33 ) años, con predominio del sexo femenino (4.5:1); originarios en su totalidad de la Ciudad de México (Tabla 2). De todos los casos estudiados fue el género femenino el que dio un mayor número de casos positivos para IRA (81.81\%), mientras que el género masculino correspondió al $18.18 \%(p<0.05)$. El
Tabla 2. Resultados demográficos y virus aislados en casos de infección respiratoria por RT-PCR

\begin{tabular}{|l|c|c|}
\hline Rango de edad & Frecuencia & $\%$ \\
\hline $15-25$ & 3 & 14 \\
\hline $26-40$ & 3 & 14 \\
\hline $41-55$ & 6 & 27 \\
\hline $56-70$ & 6 & 27 \\
\hline $71-90$ & 4 & 18 \\
\hline Total & 22 & 100 \\
\hline Tipo de virus & Frecuencia & $\%$ \\
\hline MPVh & 10 & 45.6 \\
\hline PIV 2h & 1 & 4.5 \\
\hline VSRh, MPVh & 1 & 4.5 \\
\hline VSRh, MPVh, PIV 2h & 3 & 13.7 \\
\hline VSRh, PIV 2h & 1 & 4.5 \\
\hline VSRh, MPVh & 1 & 4.5 \\
\hline VSRh-B & 5 & 22.7 \\
\hline Total & 22 & 100 \\
\hline MPVh: metapneumovirus humano; PIV 2h: parainfluenza 2 humano; VSRh: virus sincicial \\
respiratorio humano.
\end{tabular}

análisis por RT-PCR de punto final arrojó que $45.45 \%$ de los especímenes fueron positivos para MPVh; un $22.72 \%$ para VSRh y solo 1 caso $(4.52 \%)$ para PIV $2 \mathrm{~h}$. Hubo coinfección en el $27.27 \%$ de los pacientes.

\section{Etapa II. Correlación clínica}

Se correlacionaron los diferentes signos y síntomas que presentaron los pacientes estudiados. Dados los resultados, es posible aseverar que la presencia individual de MPVh, como agente causal de IRA, es responsable del mayor porcentaje de pacientes que presentaron manifestaciones clínicas; sin embargo, la coinfección VSRh y PIV 1-3h reveló una presentación clínica más grave (estridor laríngeo y disnea, 67 y $40 \%$ respectivamente) (Tabla 3 ).

Por otra parte, el VSRh fue el segundo agente más frecuente, así como el principal responsable de estertores $(40 \%)$, sibilancias $(40 \%)$, tos $(31 \%)$, cefalea $(15 \%)$ y ataque al estado general $(25 \%)$. Ahora bien, en el caso de las coinfecciones, principalmente en las cuales se identificaron los tres agentes virales, se encontró asociación con estridor laríngeo, sibilancias, disnea, fiebre, estertores, odinofagia, amigdalitis y congestión nasal. 
Tabla 3. Frecuencia de síntomas y signos de acuerdo a tipo de infección

\begin{tabular}{|c|c|c|c|c|c|c|}
\hline \multirow[t]{2}{*}{ Manifestación clínica } & \multicolumn{6}{|c|}{ Infección vital (\%) } \\
\hline & $\begin{array}{c}\text { Coinfección } \\
\text { (VSRh, MPVh, PIV 2h) }\end{array}$ & $\begin{array}{l}\text { Coinfección } \\
\text { (VSRh, MPVh) }\end{array}$ & $\begin{array}{l}\text { Coinfección } \\
\text { (hRSV, hPIV2) }\end{array}$ & VSR-B & MPVh & PIV $2 \mathrm{~h}$ \\
\hline $\begin{array}{l}\text { Síntomas } \\
\text { Fiebre } \\
\text { Cefalea } \\
\text { Ataque al estado general } \\
\text { Tos } \\
\text { Disnea } \\
\text { Cianosis }\end{array}$ & $\begin{array}{c}50 \\
15 \\
0 \\
13 \\
40 \\
50\end{array}$ & $\begin{array}{c}0 \\
8 \\
12 \\
6 \\
20 \\
50\end{array}$ & $\begin{array}{c}0 \\
8 \\
13 \\
0 \\
20 \\
0\end{array}$ & $\begin{array}{c}0 \\
15 \\
25 \\
31 \\
20 \\
0\end{array}$ & $\begin{array}{c}50 \\
46 \\
50 \\
50 \\
0 \\
0\end{array}$ & $\begin{array}{l}0 \\
8 \\
0 \\
0 \\
0 \\
0\end{array}$ \\
\hline $\begin{array}{l}\text { IVRA } \\
\text { Odinofagia } \\
\text { Rinorrea hialina } \\
\text { Congestión nasal } \\
\text { Amigdalitis } \\
\text { Estridor laríngeo }\end{array}$ & $\begin{array}{c}9 \\
17 \\
15 \\
0 \\
67\end{array}$ & $\begin{array}{c}18 \\
12 \\
8 \\
20 \\
0\end{array}$ & $\begin{array}{l}0 \\
6 \\
8 \\
0 \\
0\end{array}$ & $\begin{array}{c}9 \\
18 \\
15 \\
20 \\
0\end{array}$ & $\begin{array}{l}64 \\
41 \\
46 \\
60 \\
33\end{array}$ & $\begin{array}{l}0 \\
6 \\
8 \\
0 \\
0\end{array}$ \\
\hline $\begin{array}{l}\text { IVRB } \\
\text { Estertores } \\
\text { Sibilancia }\end{array}$ & $\begin{array}{l}20 \\
40\end{array}$ & $\begin{array}{c}20 \\
0\end{array}$ & $\begin{array}{l}0 \\
0\end{array}$ & $\begin{array}{l}40 \\
40\end{array}$ & $\begin{array}{l}20 \\
20\end{array}$ & $\begin{array}{l}0 \\
0\end{array}$ \\
\hline Dolor abdominal & 25 & 25 & 0 & 0 & 25 & 25 \\
\hline
\end{tabular}

IVRA: infección vías respiratorias altas; IVRB: infección vías respiratorias bajas.

\section{Discusión}

Al igual que en todo el mundo, en México las infecciones respiratorias son un grave problema de salud. Consideramos que conocer con mayor detalle los agentes virales causantes de IRA fortalecerán de alguna manera los esfuerzos que se han realizado para disminuir estas infecciones en nuestro país.

En una era de amenazas por enfermedades transmisibles emergentes y reemergentes no se debe subestimar la importancia de las medidas de prevención, control, diagnóstico y tratamiento de infección en los entornos de atención de la salud para evitar la aparición y el incremento de brotes. Dentro de tales preocupaciones se reconoce que aunque en los niños se requiere hacer una eficiente detección de virus como metapneumovirus humano y coronavirus humano, ello también tiene que ser extendido hacia pacientes adultos ${ }^{6,12}$. Hoy en día, la detección molecular y la caracterización de los patógenos virales presentes en las enfermedades respiratorias es uno de los desafíos más importantes relacionados con las enfermedades infecciosas.

En el presente estudio pudimos evaluar el papel de los agentes virales en las IRA en una población adulta ambulante perteneciente a una clínica de segundo nivel de la Ciudad de México; en donde se evidenció una alta presencia de los VR «poco comunes", tales como VSRh, MPVh y PIV $2 \mathrm{~h}$; debemos mencionar que la presencia de dichos patógenos es indistinguible entre sí, siendo solo diferenciada mediante la magnitud de los síntomas clínicos.

Actualmente, la identificación del agente etiológico viral en infecciones respiratorias se realiza por métodos tradicionales como aislamiento viral en cultivo celular e inmunofluorescencia directa o indirecta en muestras clínicas. No obstante, el uso de estas técnicas presenta varias desventajas, entre ellas: a) requieren de varios días o semanas para el aislamiento en cultivos celulares; b) diferencias en la actividad citopática, lo que conlleva al enmascaramiento de virus menos citopáticos y/o de lento crecimiento; c) escasa sensibilidad/especificidad; d) falsos negativos cuando se utiliza la técnica de inmunofluorescencia; e) el aislamiento viral depende del estado de la muestra, y f) contaminación bacteriana durante la toma de la muestra. Por tal motivo, se considera necesaria la implementación de estrategias de diagnóstico viral, como las utilizadas en este estudio, para mejorar la comprensión y manejo de las IRA en este tipo de población estudiada.

Los resultados obtenidos demuestran que a partir del diseño de oligonucleótidos propios se puede lograr una buena identificación adecuada del género paramixovirus. Además, al asociar estos resultados con el cuadro clínico presentado en cada caso, simbolizan una herramienta en pro del diagnóstico viral especifico; esto tuvo semejanza a lo obtenido en otros 
estudios de tipo epidemiológico, en donde la enfermedad originada por VPMh está asociada con IRA y EPOC en adultos ${ }^{13}$.

Adicionalmente, al igual que en reportes previos, se destacó que en casos de coinfecciones virales las manifestaciones clínicas relacionadas a IRA (tos, congestión nasal, rinorrea) tienden a asumir una presentación sobre expresada ${ }^{14}$.

A pesar de las limitaciones de este estudio, los resultados aquí presentados son de sumo interés, ya que la aplicación de una herramienta molecular, como la RT-PCR de punto final, parece brindar la posibilidad de llevar a cabo la tipificación de los agentes virales responsables de la infección respiratoria aguda; pudiendo constituir un apoyo al diagnóstico clínico. Asimismo, el uso de la presente metodología puede contribuir a proporcionar información para los programas nacionales de vigilancia epidemiológica sobre los virus circulantes estacionalmente en nuestro país y evaluar la aparición de variantes virales que pudieran tener relevancia clínica; permitiendo dirigir la aplicación de medidas preventivas específicas para el control, diagnóstico, pronóstico y tratamiento de estas infecciones, tanto en el primer como en el segundo nivel de atención institucional.

\section{Conclusiones}

Se considera importante realizar el diagnóstico rápido de la IRA, ya que esto implica el tratamiento temprano, así como las medidas sanitarias que impidan su dispersión a ámbitos hospitalarios y, de forma más importante, limitar el uso innecesario de antibióticos.

Según los resultados obtenidos del diagnóstico viral diferencial en el presente estudio, los VR del tipo paramixovirus se mantienen en circulación en población adulta; además, pueden estar presentes a manera de coinfecciones, lo cual empeora el cuadro clínico.

\section{Agradecimientos}

El presente trabajo de investigación fue apoyado por el programa E015 Investigación y Desarrollo Tecnológico en Salud ISSSTE y DGAPA-UNAM IN224316 y PE211115. Los autores agradecen al Dr. José Vicente Rosas Barrientos su asesoría científica, a la Q.F.B. Karla Zárate Conde, María José Chávez Barajas, Daniel Alejandro Pérez Ayala e Ilse Joana Camacho Aguilar su apoyo técnico.

\section{Bibliografía}

1. Pio A, Leowski J, Luelmo F. Programa de la Organización Mundial de la Salud de infecciones respiratorias agudas en la infancia. Bol Of Sanit Panam. 1984;96(4):283-93.

2. Secretaria de Salud. [internet] Anuario Estadístico 2016. Epidemiología. Disponible en www.epidemiologia.salud.gob.mx/anuario/ html/anuarios.

3. Wylie KM. The virome of the human respiratory tract. Clin Chest Med. 2017;38:11-9.

4. Ogra PL. Respiratory syncytial virus: the virus, the disease and the immune response. Paediatr Respir Rev. 2004;5(Suppl A):S119-26.

5. Colosia AD, Yang J, Hillson E, Mauskopf J, Copley-Merriman C Shinde V, et al. The epidemiology of medically attended respiratory syncytial virus in older adults in the United States: A systematic review. PLoS One. 2017;12:e0182321.

6. Volling C, Hassan K, Mazzulli T, Green K, Al-Den A, Hunter P, et al. Respiratory syncytial virus infection-associated hospitalization in adults: a retrospective cohort study. BMC Infect Dis. 2014;14:665.

7. Mackay IM, Jacob KC, Woolhouse D, Waller K, Syrmis MW, Whiley DM, et al. Molecular assays for detection of human metapneumovirus. J Clin Microbiol. 2003;41:100-5.

8. Fields B, Knipe DM, Howley PM. Virology. Philadelphia: Wolters Kluwer/ Lippincott Williams \& Wilkins; 2007.

9. Romero Feregrino R, Romero Feregrino R, Magaña IM, Romero CabeIlo R, González Saldaña NJ. Diagnóstico de Metapneumovirus humano. Revista de Enfermedades Infecciosas en Pediatría. 2013;26:256-69.

10. Visseaux B, Burdet C, Voiriot G, Lescure FX, Chougar T, Brugière O, et al. Prevalence of respiratory viruses among adults, by season, age, respiratory tract region and type of medical unit in Paris, France, from 2011 to 2016. 2017;12:e0180888.

11. Instituto Mexicano del Seguro Social. Guía de práctica clínica de diagnóstico y tratamiento de bronquiolitis aguda en niñas/niños y en el Primer Nivel de Atención. México; 2015.

12. Dayakar S, Pillai HR, Thulasi VP, Nair RR. Development of a multiplex RT-PCR for simultaneous diagnosis of human metapneumovirus (HMPV) and human respiratory syncytial virus (HRSV) from clinical specimens. 2016;27:375-81.

13. Stockton J, Stephenson I, Fleming D, Zambon MJ. Human metapneumovirus as a cause of community-acquired respiratory illness. 2002;8:897.

14. Martinello RA, Esper F, Weibel C, Ferguson D, Landry ML, Kahn JS. Human metapneumovirus and exacerbations of chronic obstructive pulmonary disease. 2006;53:248-54. 